\title{
Long Term Follow-Up of ABO Incompatible Kidney Transplantation-A Study from India
}

\author{
Rajan Ravichandran', Subramanian Kannan ${ }^{2}$ \\ ${ }^{1}$ Miot Institute of Nephrology, Miot Hospitals, Chennai, India \\ ${ }^{2}$ Madras Institute of Nephrology Research Pvt. Ltd., Chennai, India \\ Email: ravidoc55@yahoo.co.in, mionresearch@yahoo.com
}

Received 9 March 2016; accepted 26 March 2016; published 29 March 2016

Copyright (C) 2016 by authors and OALib.

This work is licensed under the Creative Commons Attribution International License (CC BY). http://creativecommons.org/licenses/by/4.0/

(c) (i) Open Access

\begin{abstract}
Background: There is a need to do ABO incompatible kidney transplantation in a country like India where deceased donor transplantation is not common. More than $\mathbf{9 0 \%}$ transplantations are done with living donors. Although short term results have been reported from 4 centers, there is paucity of data on long term follow-up of such patients. Methods: The data of 18 patients who had undergone $\mathrm{ABO}$ incompatible transplantation exceeding a follow-up of 12 months up to 66 months were analyzed. It was compared with the data of compatible kidney transplantation during the same period. Results: In both the groups, there was a similar incidence of urinary tract infection and gastroenteritis with complete recovery. Tuberculosis was successfully treated in 2 patients who had undergone $\mathrm{ABO}$ incompatible transplantation. The graft function was excellent with an average follow-up period of $\mathbf{3 8 . 2}$ months. There was no patient mortality during the follow-up period. Conclusion: The long term outcome of $\mathrm{ABO}$ incompatible kidney transplantation is similar to compatible kidney transplantation despite the increased incidence of short term complications.
\end{abstract}

\section{Keywords}

ABO Incompatible Kidney Transplantation, India, Long Term

Subject Areas: Nephrology

\section{Introduction}

ABO (Blood group) incompatible kidney transplantations are getting popular all over the world due to shortage of organs. Several centers have reported excellent short term and long term results [1]-[6]. One of the biggest concerns following such transplantations in a country like India is the possible increased incidence of infections. Even following compatible kidney transplantations, a high incidence of Tuberculosis, gastroenteritis and Urinary Tract Infections (UTI) has been reported [7]-[9]. Success of ABO incompatible kidney transplantation de- 
pends on desensitization protocol with Rituximab, plasma exchange and higher immunosuppression in the perioperative period [10]. This can pose a higher risk for both short term and long term infections [11]. We started our ABO incompatible kidney transplantation in 2010 and reported the short term outcome in 13 patients with loss of graft in 2 due to antibody mediated rejections [12]. Similarly, Virani et al. [13] and P. K. Jha et al. [14] have reported a successful short term outcome from Mumbai and Delhi. The present study is an analysis of the data of patients who have crossed 12 months post successful ABO incompatible kidney transplantation up to a period of 66 months with special reference to kidney function and infections.

\section{Materials and Methods}

Data were collected from 18 patients who had undergone ABO incompatible kidney transplantation between 2010 and 2014. Only those patients who had crossed a follow up of more than 12 months were included in the study. ABO compatible kidney transplantation recipients during the same period were taken as controls. All transplantations were done in a single center with necessary permission from the hospital committee. Informed consent was taken from all the patients. Pre operative work up was similar in both the groups except that the incompatible group had monitoring of Anti-A, Anti-B titers by the gel card method. Patients on the incompatible group were hospitalized 7 to 10 days prior to surgery. The desensitization protocol of Tanabe [6] was followed. Rituximab (Roche) was given $200 \mathrm{mcg}$ on day -7. Patients underwent plasma exchange $40 \mathrm{ml} / \mathrm{kg}$ body weight on alternate days. The average number of plasma exchanges done was 4. Triple immunosupression was started with steroids, Mycophenolate and Tacrolimus. Transplantation was done if the titer dropped to equal or less than 1:16. Basiliximab was given on day 1 and 4 . The control group also received induction therapy with Basiliximab and triple immunosuppression with steroids, Mycophenolate and Tacrolimus. Both the group received post operative prophylaxis with Cotrimoxazole. The ABO incompatible group additionally received Valganciclovir for 3 months. Anti-A and Anti-B titers were monitored daily for the $1^{\text {st }}$ week and alternate days in the $2^{\text {nd }}$ week. Patients were followed up weekly once for the first 3 months, once in 15 days for 6 months and once a months there after (Table 1).

Table 1. Demographics and long term complications in both compatible and incompatible transplantations.

\begin{tabular}{lcc}
\hline & ABO incompatible group & ABO compatible group \\
\hline Total no. of patients who exceeded follow up of 12 months & 18 & 21 \\
Average follow up (in months) & 37.6 (17 to 66 months) & 38.9 (18 to 65 months) \\
Average age (in years) & 36.7 (13 to 57 years) & 42.1 (17 to 64 years) \\
Male & 11 & 17 \\
Female & 7 & 4 \\
Average serum creatinine at the end of follow-up & 1.3 mg/dl (0.8 to 2.0$)$ & 4.7 to 1.9$)$ \\
Complications & & 1 \\
Gastroenteritis & 4 & 5 \\
CMV infection & 1 & Nil \\
UTI & 4 & 1 \\
Tuberculosis & 2 & 1 \\
NODM & 2 & 1 \\
Epididymo-orchitis & Nil & Nil \\
Gangrene & Nil & 6 \\
Mortality & Nil & 5 \\
Hospitalization & 5 & 1 \\
\hline
\end{tabular}

CMV: Cytomegalovirus, UTI: Urinary Tract Infection, NODM: New Onset Diabetes Mellitus. 


\section{Results}

\subsection{ABO Incompatible Group}

The total number of patients who exceeded follow up of 12 months was 18. Range of follow up was 17 months to 66 months (Average 37.6). Age range was 13 to 57 years (Average 36.7). Number of male patients was 11 and number of female patients was 7. Serum creatinine range at the end of follow-up was 0.8 to $2.0 \mathrm{mg} / \mathrm{dl}$ (Average $1.3 \mathrm{mg} / \mathrm{dl}$ ). 11 patients had O blood group, 4 patients had A blood group and 3B blood group. In 8 patients parents were the donor, in 8 spouse, in 1 sibling and in 1 unrelated. 4 patients were diabetic. Underlying kidney disease was, Focal segmental glomerulosclerosis (FSGS) in 1, Reflux Nephropathy in 2, IgA Nephropathy in 3, Diabetic Nephropathy in 3, SLE in 1 and rest 8 had contracted kidneys.

\subsection{Complications Post 12 Months}

4 patients had gastroenteritis which required hospitalization and hydration. Average period of hospitalization was 4.5 days. No specific organism was grown in the feces. They recovered well without any complication. One patient developed Cytomegalovirus (CMV) infection with fever, leucopenia 18 months after the transplant and was successfully treated with Valganciclovir and switching of Mychophenolate to Azathioprine. 4 patients had UTI, out of which 3 of them required hospitalization and parental antibiotics. 2 patients continued to have recurrent UTI which settled after 6 months. 2 of the patient had infection due to klebsiella and 2 had due to E.coli. 1 patient 14 months after transplantation developed miliary tuberculosis and was successfully treated with a combination of 4 drugs for a period of 12 months. Another patient developed tuberculous pleural effusion 3 years post transplantation which also responded to treatment. 2 patients developed New Onset Diabetes Mellitus (NODM). There was no mortality amongst these patients and the recovery from infection was complete.

\subsection{Graft Function}

1 patient had serum Creatinine of $2.0 \mathrm{mg} / \mathrm{dl}$ at the end of 66 months. He had received kidney from his father who was 64 years of age at the time of donation. The lowest creatinine he achieved was $1.5 \mathrm{mg} / \mathrm{dl}$ in the immediate perioperative period. The average creatinine in the group was $1.3 \mathrm{mg} / \mathrm{dl}$ (range 0.8 to $2.0 \mathrm{mg} / \mathrm{dl}$ ). 1 patient had kidney biopsy for protienuria and worsening of kidney function and the biopsy showed FSGS. This kidney function and protienuria stabilized with Angiotensin Receptor Blockers (Serum Creatinine $1.6 \mathrm{mg} / \mathrm{dl}$ and protein loss $1.2 \mathrm{gm} / \mathrm{kg} /$ day in the last follow-up). There was no graft loss between 12 months to 66 months of follow-up.

\subsection{ABO Compatible Group}

Total number of patients followed was 21. Period of follow-up was 18 to 65 months (average 38.9). Age range was 17 to 64 years (Average 42.1). Male patients were 17 and female 4.7 patients had O blood group, 5 A blood group, $1 \mathrm{AB}$ blood group and $8 \mathrm{~B}$ blood group. Serum creatinine ranged between 0.7 to $1.9 \mathrm{mg} / \mathrm{dl}$ (average 1.3 $\mathrm{mg} / \mathrm{dl}$ ). In 9 patients parents were the donor, in 9 spouse, in 1 sibling, in 1 daughter and in 1 unrelated. 4 patients were diabetic. Underlying kidney disease was Diabetic Nephropathy in 3, Reflux Nephropathy in 2, IgA Nephropathy in 5 and rest had contracted kidneys.

\subsection{Complications Post 12 Months}

5 patients had UTI with 1 patient developing Epididymo-orchitis also. 4 patients required hospitalization and IV antibiotics. All of them recovered well except 1 patient who developed persistent impaired kidney function with serum creatinine increasing from 1.2 to $1.5 \mathrm{mg} / \mathrm{dl} .4$ patients had gastroenteritis requiring hospitalization and hydration. 1 patient presenting as gastroenteritis had CMV infection which was successfully treated with Valgancyclovir and reduction of immunosuppression. 1 diabetic patient developed gangrene due to peripheral vascular disease. There was no mortality in any of the patients.

\subsection{Graft Function}

Graft function was impaired in 1 patient who had serum creatinine of $1.9 \mathrm{mg} / \mathrm{dl}$, the biopsy showing chronic allograft nephropathy. The average serum creatinine in the group was $1.3 \mathrm{mg} / \mathrm{dl}$ (range 0.7 to $1.9 \mathrm{mg} / \mathrm{dl}$ ). 2 pa- 
tients developed proteinuria. The biopsy showed chronic allograft nephropathy. There was no graft loss in the group.

\section{Discussion}

ABO incompatible kidney transplantation is being done in several countries with successful results [1]-[6]. Shortage of organs makes it necessary to explore the possibility of such transplants in a developing country also. In a country like India, about 200,000 patients require renal replacement therapy every year and only about 5000 kidney transplantations are done [15]. Majority of these transplantations are done with living donors. Deceased donor transplantation is just picking up in some of the states in India. ABO incompatible transplantation is being done sporadically throughout the country. But only a handful of centers do on a regular basis. This is because of two reasons. a) A reluctance in the part of the Nephrologists due to possible increased incidence of infections and antibody mediated rejections in these patients. b) The cost of such transplantation is almost double that of regular kidney transplantation.

Infections play a major role in the long term outcome of compatible renal transplantation. GT John et al. reported a $13.3 \%$ incidence of tuberculosis in a follow-up of 13.9 years. Out of 166 patients who developed tuberculosis, 53 died and 17 patients had co-existing fungal, nocardia or CMV infections [8]. Bhadauria et al. reported an incidence of diarrhea in $8.9 \%$ of the recipients, $28 \%$ of them due to Cryptosporidiosis [7]. Iqbal et al. reported 33\% of UTI [9]. In 77\% of the patients, it occurred within first 2 months and the rest during the long term follow-up.

The success of $\mathrm{ABO}$ incompatible kidney transplantation depends on a desensitization protocol in the perioperative period to bring down the blood group titers [10]. This could involve administration of Rituximab in varying doses, plasma exchange with or without Intravenous immunoglobulin, double filtration plasmapheresis on alternate days or immunoadsorption till the titer drop to acceptable levels. Additional induction is also given either with Basiliximab or Anti-thymocyte globulin. Although several centers have reported a successful short term outcome using various protocols, there has been an increased incidence of perioperative bleeding episodes, infections and antibody mediated rejections [2] [6] [10] [11] [16]. Normally after 4 weeks, the immunosuppression protocol is similar in both ABO incompatible and compatible kidney transplantations. The effect of Rituximab lasts for almost 6 months. Hence, there is a concern about the long term incidence of infections in these patients. Several centers have reported excellent outcomes with a follow-up period exceeding 10 years both in terms of complications and graft function [1] [3] [6]. In the present study, the data have been collected from 18 patients who had crossed 1 year after a successful ABO incompatible kidney transplantation. They were compared to a similar group of compatible patients who had undergone transplantation during the same period. The graft function is similar in the average follow-up of 38.2 months in both the groups. There were no episodes of late rejections in both the groups. However 3 patient developed chronic allograft nephropathy with mild deterioration of renal function in the compatible group. 1 patient developed recurrence of FSGS in the incompatible group. The rate of infection after 1 year from transplantation is also very similar in both the groups. Gastroenteritis had the highest incidence followed by UTI. Tuberculosis occurred in 2 patients in the incompatible group with one of them developing a miliary form. In both the groups, patients could be successfully treated for the infections. There was no mortality in both the groups and the graft survival was also $100 \%$ between the timeframe of 17 to 66 months. The average cost of compatible kidney transplantation was 7500 USD. The cost in the incompatible group was 15,000 USD. Our results should give confidence to more transplant centers in developing countries to offer $\mathrm{ABO}$ incompatible kidney transplantation on a regular basis. The study has limitation in terms of low number of patients and an average follow-up of 38.2 months only.

\section{References}

[1] Wilpert, J., Fischer, K., Pisarski, P., Wiech, T., Daskalakis, M., Ziegler, A., Neumann-Haefelin, E., Drognitz, O., Emmerich, F., Walz, G. and Geyer, M. (2010) Long-Term Outcome of ABO-Incompatible Living Donor Kidney Transplantation Based on Antigen-Specific Desensitization. An Observational Comparative Analysis. Nephrology Dialysis Transplantation, 25, 3778-3786. http://dx.doi.org/10.1093/ndt/gfq229

[2] Montgomery, J., Berger, J., Warren, D., James, N., Montgomery, R. and Segev, D. (2012) Outcomes of ABO-Incompatible Kidney Transplantation in the United States. Transplantation, 1. http://dx.doi.org/10.1097/TP.0b013e318245b2af 
[3] Uchida, J., Kuwabara, N., Machida, Y., Iwai, T., Naganuma, T., Kumada, N. and Nakatani, T. (2012) Excellent Outcomes of ABO-Incompatible Kidney Transplantation: A Single-Center Experience. Transplantation Proceedings, 44, 204-209. http://dx.doi.org/10.1016/j.transproceed.2011.11.006

[4] Oettl, T., Halter, J., Bachmann, A., Guerke, L., Infanti, L., Oertli, D., Mihatsch, M., Gratwohl, A., Steiger, J. and Dickenmann, M. (2008) ABO Blood Group-Incompatible Living Donor Kidney Transplantation: A Prospective, Single-Centre Analysis Including Serial Protocol Biopsies. Nephrology Dialysis Transplantation, 24, 298-303. http://dx.doi.org/10.1093/ndt/gfn478

[5] Lipshutz, G. (2011) ABO Blood Type-Incompatible Kidney Transplantation and Access to Organs. Archives of Surgery, 146, 453. http://dx.doi.org/10.1001/archsurg.2011.40

[6] Tanabe, K. (2007) Japanese Experience of ABO-Incompatible Living Kidney Transplantation. Transplantation, 84, S4-S7. http://dx.doi.org/10.1097/01.tp.0000296008.08452.4c

[7] Bhadauria, D., Goel, A., Kaul, A., Sharma, R., Gupta, A., Ruhela, V., Gupta, A., Vardhan, H. and Prasad, N. (2015) Cryptosporidium Infection after Renal Transplantation in an Endemic Area. Transplant Infectious Disease, 17, 48-55. http://dx.doi.org/10.1111/tid.12336

[8] John, G., Shankar, V., Abraham, A., Mukundan, U., Thomas, P. and Jacob, C. (2001) Risk Factors for Post-Transplant Tuberculosis. Kidney International, 60, 1148-1153. http://dx.doi.org/10.1046/j.1523-1755.2001.0600031148.x

[9] Iqbal, T., Naqvi, R. and Akhter, S.F. (2010) Frequency of Urinary Tract Infection in Renal Transplant Recipients and Effect on Graft Function. Journal of Pakistan Medical Association, 60, 826-829.

[10] Shin, M. and Kim, S. (2011) ABO Incompatible Kidney Transplantation-Current Status and Uncertainties. Journal of Transplantation, 2011, 1-11. http://dx.doi.org/10.1155/2011/970421

[11] Habicht, A., Broker, V., Blume, C., Lorenzen, J., Schiffer, M., Richter, N., Klempnauer, J., Haller, H., Lehner, F. and Schwarz, A. (2011) Increase of Infectious Complications in ABO-Incompatible Kidney Transplant Recipients-A Single Centre Experience. Nephrology Dialysis Transplantation, 26, 4124-4131. http://dx.doi.org/10.1093/ndt/gfr215

[12] Ravichandran, R., Kanakaraj, A., Shakthivel, A. and Srinivas, C. (2012) ABO Incompatible Kidney TransplantationA Single Center Experience. Indian Journal of Transplantation, 6, 103-106. http://dx.doi.org/10.1016/j.ijt.2012.10.002

[13] Virani, Z., Bhagat, A., Rajput, P., Shah, B., Pagar, S., Bala, S. and Dawane, V. (2014) Our Initial Experience with ABO-Incompatible Transplant. Indian Journal of Transplantation, 8, 132-133. http://dx.doi.org/10.1016/j.ijt.2014.12.021

[14] Jha, P.K., Bansal, S.B., Sethi, S.K., et al. (2015) ABO-Incompatible Renal Transplantation in Developing WorldCrossing the Immunological (and Mental) Barrier. Indian Journal of Nephrology, 26, 113-118. http://www.indianjnephrol.org/preprintarticle.asp?id=159557

[15] Jha, V. (2013) Current Status of End-Stage Renal Disease Care in India and Pakistan. Kidney International Supplements, 3, 157-160. http://dx.doi.org/10.1038/kisup.2013.3

[16] Tobian, A., Shirey, R., Montgomery, R., Cai, W., Haas, M., Ness, P. and King, K. (2010) ABO Antibody Titer and Risk of Antibody-Mediated Rejection in ABO-Incompatible Renal Transplantation. American Journal of Transplantation, 10, 1247-1253. http://dx.doi.org/10.1111/j.1600-6143.2010.03103.x 